\title{
Aortoenteric Fistula Causing Syncope and Gastrointestinal Bleeding
}

\author{
Aureliu Grasun' (D), Hector Alonso Valle ${ }^{1}$ (D), María Andrés Gómez' (D), Luis Prieto Lastra' (iD, Pablo Garmilla Ezquerra' (D), \\ Francisco Manuel Mateos Chaparro' (D), Nuria Odriozola Romillo ${ }^{1}$ (D), Elena Grasun ${ }^{1}$ (D), Sergio Rubio Sánchez ${ }^{1}$ (D), Luis Iglesias Oliva' (D), \\ Patricio Alberto Seabrook Maggio² (D), Ricardo Jauregui Solorzano² (D) \\ 'Department of Emergency, The Marqués De Valdecilla University Hospital, Santander, Spain \\ ${ }^{2}$ Department of Intensive Care Unit, The Marqués De Valdecilla University Hospital, Santander, Spain
}

\section{Cite this article as: Grasun A, Valle HA, Gómez MA, Lastra LP, Ezquerra PG, Mateos Chaparro FM, et al. Aortoenteric Fistula Causing} Syncope and Gastrointestinal Bleeding. Eurasian J Emerg Med. 2018; 17: 36-8.

\begin{abstract}
Aortoenteric fistula (AEF) is an uncommon and emergency clinical condition of intra-abdominal bleeding. Early diagnosis can be achieved by computed tomography and endoscopy exploration. Surgical reconstruction is an urgent and multidisciplinary treatment and is recommended after the surgical intervention. A 76-year-old man with a history of abdominal aortic aneurysm reconstruction presented to the emergency department with syncope and suspected upper gastrointestinal (Gl) bleeding, which began approximately $30 \mathrm{~min}$ before. In the hospital, the patient's condition worsened and he developed cardiac arrest due to massive Gl bleeding. The advanced cardiopulmonary resuscitation was initiated and with suspected active aortoenteric communication, an urgent laparoscopy was immediately performed.
\end{abstract}

Keywords: Aorto-enteric fistula, syncope, gastrointestinal bleeding

\section{Introduction}

The aortoenteric fistula (AEF) is a direct communication between the aorta and intestine (1). There are two forms of AEFs: 1) Primary, which usually occur due to the erosion of an abdominal aortic aneurysm (AAA) into the intestine and 2) Secondary, which are caused by reconstructive procedures on the abdominal aorta (2). The incidence of primary AEF ranges from $0.1 \%$ to $0.8 \%$ and that of secondary from $0.4 \%$ to $2.4 \%(1,3)$. The mortality rate after surgical treatment of secondary AEFs is from $14 \%$ to $70 \%$ (1).

\section{Case Presentation}

In January 2017, a 76-year-old man was transported by the Emergency Medical Services to our emergency department (ED) after experiencing an attested episode of syncope at home approximately 30 min before. His past medical history included arterial hypertension, chronic pulmonary obstructive disease, hiatus hernia, and a gastric ulcer. At the beginning of 2015, he was diagnosed with Leriche's syndrome and later in September 2015 he underwent a bypass aortobifemoral without initial complications. Furthermore, in January 2016,

ORCID IDs of the authors: A.G. 0000-0003-4864-1475; H.A.V. 0000-0002-6070-8874; M.A.G. 0000-0001-7485-4939; L.P.L. 0000-0002-3057617X; P.G.E. 0000-0002-3770-164X; F.M.M.C. 0000-0002-6915-7458; N.O.R. 0000-0001-8742-6140; E.G. 0000-0001-5871-5920; S.R.S. 00000001-7761-0067; L.I.O. 0000-0002-0912-5286; P.A.S.M. 0000-0003-1513-7176; R.J.S. 0000-0003-4678-5686. 


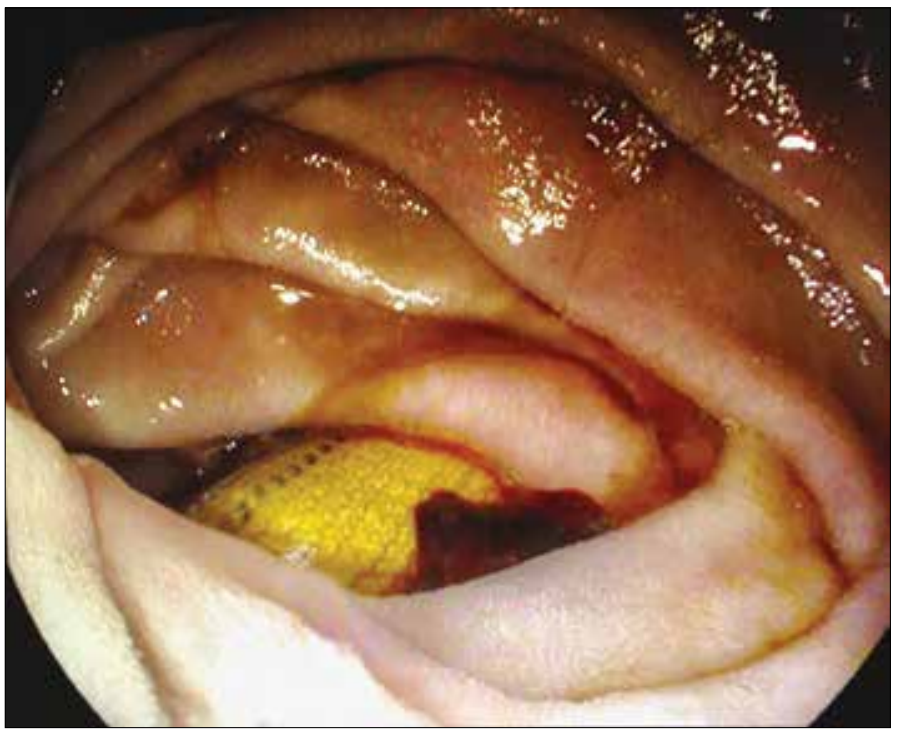

Figure 1. Upper digestive endoscopy: the Dacron prosthesis in the intestinal lumen is objectified

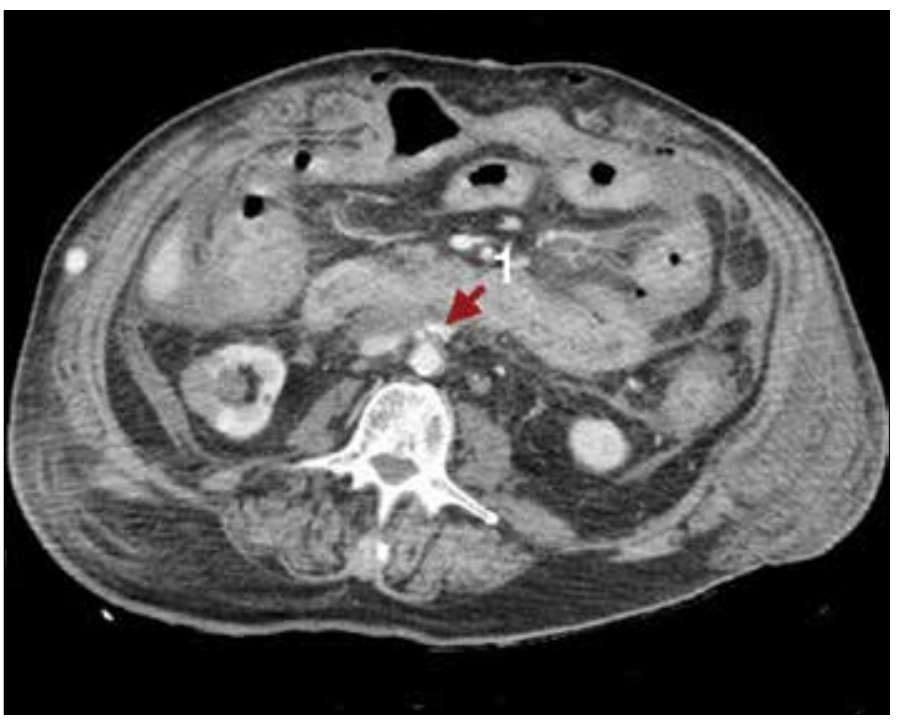

Figure 2. CT abdominal scan with extravasation of IV contrast (1) in relation to active bleeding around the surgical drainage catheter

the patient had a superficial femoral artery obstruction, and a left posterior tibial femoral bypass was performed. During the transfer to the hospital, the patient presented a self-limiting episode of hematemesis with fresh red blood. Upon arrival in the ED, the patient remained hemodynamically stable. Clinically, he complained of general sickness, weakness, and dizziness. The primary physical examination revealed paleness of the skin and tachypnea with simultaneous tachycardia; however, the remaining examination was unremarkable. The patient was connected to continuous cardiac monitoring and a complete analytical test was performed. Approximately 15 minutes after arriving at the hospital, the patient's condition worsened clinically with intense dizziness, profuse sweating, and arterial hypotension. Thereafter, the patient presented another episode of hematemesis together with hematochezia. Against maintained arterial hypotension, despite intensive fluid therapy, jugular vein access was obtained with associated noradrenaline perfusion in the doses of up to $18 \mathrm{~mL} / \mathrm{h}$, which recovered the blood pressure and the patient was stabilized. Due to a suspicion of active upper gastrointestinal (Gl) bleeding, an endoscopy (Figure 1) was performed and bleeding was observed in the distal part of the stomach (adrenaline was injected and clips were placed). After passing through the duodenum, a foreign body with a tubular morphology was observed (Figure 1). A few minutes after performing the endoscopy, the patient presented a new episode of hematemesis, associated with progressive worsening and decreased blood pressure until cardiorespiratory arrest (pulseless electrical activity) was confirmed. Therefore, we initiated cardiopulmonary resuscitation (CPR) and proceeded to intubate the patient. The CPR lasted approximately $7 \mathrm{~min}$ until the recovery of the pulse, during which $3 \mathrm{mg}$ of adrenaline was administered. An emergent laparotomy confirmed the fistula between the bypass at the proximal level and duodenum with a dehiscent suture at that level. An axillary-bifemoral bypass with Dacron was implanted. After surgery, the patient continued to experience moderate bleeding through abdominal drainage in the context of coagulopathy, necessitating transfusion of blood products. Early signs of hypoperfusion were observed in the lower right limb after surgery, as well as signs of rhabdomyolysis, and a conservative treatment was initiated. However, finally, supracondylar amputation was necessary after the observation of established limb ischemia. Broad-spectrum antibiotic treatment with fungus coverage was used, with good clinical response at the beginning. After 7 days in the intensive care unit (ICU), the clinical situation of the patient was complicated with acute renal failure and signs of sepsis. A few days later, the patient again began to experience active bleeding from the abdominal drainage (Figure 2), and his hemodynamic status worsened terminating in death without further surgical re-intervention due to the family's wishes.

\section{Discussion}

AEF is a life-threatening and devastating cause of Gl bleeding, which can be difficult to diagnose and treat. Although it is a clinical situation infrequently seen in daily practice, it is commonly seen as a late complication of aortic reconstruction due to AAA (2). AEF is divided into 2 types: primary AEF and secondary AEF. The primary AEF occurs when a large untreated aneurysm erodes into the bordering bowel. The third portion of the duodenum, immovable retroperitoneally to the descending aorta, is the most vulnerable bowel segment to this condition $(2,3)$. The development of AEF starts with ischemia and subsequent necrosis of the intestinal wall because of repetitive traumatic pulsations of a proximate aortic aneurysm. Conditions that can albeit less commonly lead to primary AEF include syphilis, tuberculosis, mycotic infection, and collagen vascular disease, where the chronic inflammation leads to aortitis, erosion, and formation of the fistula. In the absence of treatment, the mortality scale is almost $100 \%$. With surgical intervention, survival ranges from $18 \%$ to $93 \%$. Secondary AEF occurs as a complication of a previous aortic reconstructive surgery. An estimated $80 \%$ of secondary AEF cases affect the duodenum. As a result of perigraft infection and/or the continuous pressure on the bowel from aortic pulsations, fistulas are formed $(2,4)$. Secondary AEF is a rare but well-known complication after AAA repair, occurring in $0.4 \%-1.6 \%$ of cases $(1,3)$. The most common characteristics of primary AEFs are Gl bleeding (64\%), abdominal pain (32\%), and a pulsatile abdominal mass (25\%). These 3 clinical situations are jointly present in only $10 \%$ $23 \%$ of patients. The patients with a secondary AEFs habitually present 
with Gl bleeding (80\%), sepsis (44\%), abdominal pain (30\%), back pain $(15 \%)$, groin mass $(12 \%)$, and abdominal pulsatile mass $(6 \%)(2,5)$. The diagnosis can be established based on the symptomatology and with the support of the following diagnostic tests: abdominal computed tomography (CT) with intravenous (IV) contrast, esophagogastroduodenoscopy, and arteriography. The CT scan is superior because it is less invasive, more readily available, and more expedient than other tests (2). Also, the CT scan may detect abnormal communication between the aorta and the bowel, may uncover loss of continuity of the aneurysmal wall, and may reveal air bubbles in the aneurysm wall that are pathognomonic for the existence of a fistula (2). The treatment of AEF repair is controversial. The explanation of the infected graft and perforated bypass remains the gold standard. Recent studies have suggested that in situ aortic reconstruction using antibiotic-impregnated graft, cryopreserved allograft, or autogenous vein are associated with fewer amputations, conduit failures, re-infections, and a lower rate of early and late mortalities compared to extra-anatomic bypass. Surgical treatment must be combined with antibiotic therapy, but no guidelines exist on the exact duration of treatment (4).

\section{Conclusion}

AEF is a rare but life-threatening complication occurring after endovascular AAA repair that is difficult to diagnose and treat. In patients with active $\mathrm{GI}$ bleeding and a history of aortic reconstruction, the possibility of $A E F$ must also be considered. $A E F$ requires decisive and urgent surgical management. The supporting role of emergency physicians in the ED includes optimally resuscitating the patient with fluids and blood components while initiating broad-spectrum IV antibiotics as part of sepsis management. The optimal method of management of this condition is surgery in the initial phase and later a multidisciplinary approach in ICUs.
Informed Consent: Written informed consent was obtained from patients' family who participated in this study.

Peer-review: Externally peer-reviewed.

Author Contributions: Concept - A.G., H.A.V., M.A.G., L.P.L., P.G.E., F.M.M.C., N.O.R., E.G., S.R.S., L.I.O., P.A.S.M., R.J.S.; Design - A.G., H.A.V., M.A.G., L.P.L., P.G.E., F.M.M.C., N.O.R., E.G., S.R.S., L.I.O., P.A.S.M., R.J.S.; Supervision - A.G., H.A.V., M.A.G., L.P.L., P.G.E., F.M.M.C., N.O.R., E.G., S.R.S., L.I.O., P.A.S.M., R.J.S.; Resources - A.G., H.A.V.; Materials - A.G.; Data Collection and/or Processing - A.G., M.A.G.; Analysis and/or Interpretation - A.G., H.A.V.; Literature Search - A.G., H.A.V.; Writing Manuscript - A.G.; Critical Review - A.G., H.A.V.

Conflict of Interest: No conflict of interest was declared by the authors.

Financial Disclosure: The authors declared that this study has received no financial support.

\section{References}

1. Davidovic LB, Spasic DS, Lotina SI, Kostic DM, Cinara IS, Svetkovic SD, et al. Aorto-enteric fistulas. Srp Arh Celok Lek. 2001; 129: 183-93.

2. Singh M, Koyfman A, Martinez JP. Abdominal Vascular Catastrophes. Emerg Med Clin N Am. 2016; 34: 327-39. [CrossRef]

3. Busuttil SJ, Goldstone J. Diagnosis and management of aorto-enteric fistulas. Sem Vasc Surg. 2001; 14: 302e11.

4. Chenu C, Marcheix B, Barcelo C, Rousseau H. Aorto-enteric fistula after endovascular abdominal aortic aneurysm repair: case report and review. Eur J Vasc Endovasc Surg. 2009; 37: 401-6. [CrossRef]

5. Eldrup-Jorgensen J, Hawkins RE, Bredenberg CE, Abdominal vascular catastrophes. Surg Clin North Am. 1997; 77: 1305-20. 